\title{
Spectrum of magnetic resonance imaging findings in ovarian torsion
}

\author{
Tulika Singh ${ }^{A, B, D, E}$, Nidhi Prabhakar ${ }^{A, B, E, F}$, Veenu Singla ${ }^{B, D, E}$, Rashmi Bagga ${ }^{B, D, E}$, Niranjan Khandelwal ${ }^{E, F}$ \\ Postgraduate Institute of Medical Education and Research, Chandigarh, India
}

\section{Abstract}

Purpose: Ovarian torsion is the twisting of the ovary on its vascular pedicle resulting in vascular compromise. Diagnosis of ovarian torsion is challenging in patients who have atypical clinical or ultrasound (US) findings. The objective of our study was to demonstrate the magnetic resonance imaging (MRI) features of ovarian torsion to help radiologists make a conclusive diagnosis when the clinical and US findings are unclear.

Material and methods: We retrospectively reviewed the clinical and MRI features in 10 females with surgically proven ovarian torsion, who had inconclusive clinical, US, or computed tomography findings.

Results: All patients showed a significantly enlarged ovary with size ranging from 5 to $18 \mathrm{~cm}$. 'Twisted ovarian pedicle' sign was seen in seven patients. Eight cases showed areas of haemorrhage within the ovarian stroma. Non-enhancement of ovarian stroma was observed in six patients. Seven patients showed an ipsilateral deviation of the uterus.

Conclusions: MRI features of ovarian torsion include ovarian enlargement, twisted ovarian pedicle, ovarian haemorrhage, abnormal ovarian enhancement, and ipsilateral deviation of the uterus. Awareness of these imaging features will enable the radiologist to recognise ovarian torsion and differentiate it reliably from other benign or malignant ovarian lesions.

Key words: ovary, torsion, infarction, MRI.

\section{Introduction}

Ovarian torsion is the twisting of the ovary on its vascular pedicle resulting in vascular compromise [1]. Timely identification of ovarian torsion is important because ovarian salvage is possible by surgical untwisting. Infarction and haemorrhagic necrosis occur if no surgical intervention is made. It is also essential to diagnose an infarcted ovary because an untreated infarcted ovary may lead to thrombophlebitis, infection, peritonitis, and sepsis [2].

Ovarian torsion classically presents as lower abdominal or pelvic pain in young females. Ultrasound (US) shows an enlarged ovary with peripherally displaced follicles. Colour Doppler may show absent colour flow [1]. Diagnosis of ovarian torsion is challenging in patients who have atypical clinical or US findings [3]. Magnetic resonance imaging (MRI) can act as the problem solver in complex cases. It not only helps in diagnosis of ovarian torsion but may also assist in identifying infarcted ovaries secondary to torsion.

There are limited published data available on the MRI features of ovarian torsion. The objective of our study was to demonstrate the common MRI imaging features of ovarian torsion to help radiologists make a reliable diagnosis when the clinical and US findings are unclear.

\section{Material and methods}

This study was approved by the departmental ethical committee. We retrospectively reviewed the clinical and MRI features in 10 females with surgically proven ovarian torsion. All these patients had inconclusive clinical, US or computed tomography (CT) findings, and MRI had been done to confirm the diagnosis of ovarian torsion.

MR imaging had been performed using a 1.5-Tesla MRI (Magnetom Aera, Siemens). T1-weighted images

\section{Correspondence address:}

Dr. Tulika Singh, Postgraduate Institute of Medical Education and Research, Sector-12, 160012, Chandigarh, India, e-mail: tulikardx@yahoo.com

Authors' contribution:

A Study design - B Data collection · C Statistical analysis · D Data interpretation - E Manuscript preparation · F Literature search · G Funds collection 

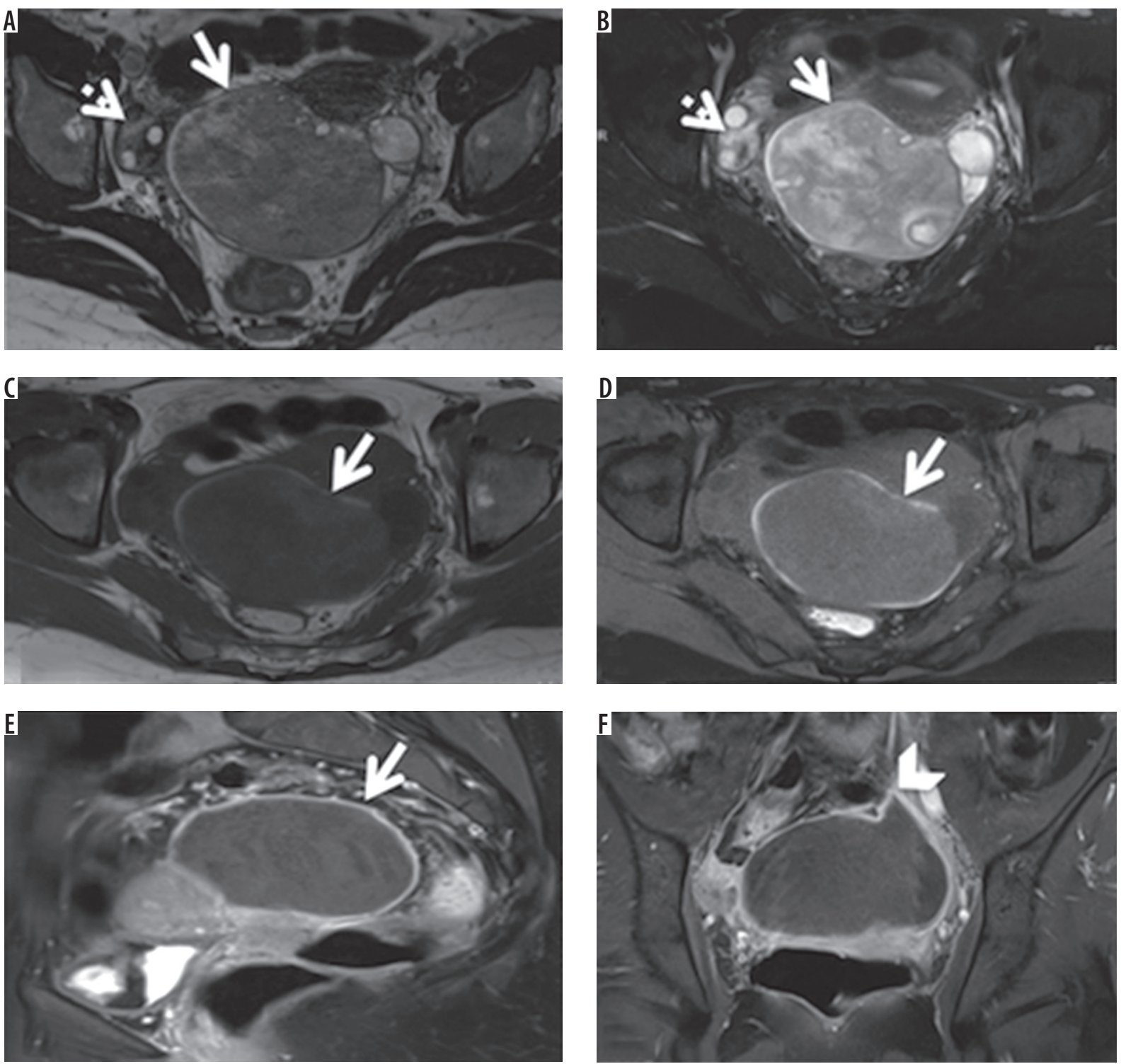

Figure 1. An 18-year-old female who complained of dull pain in lower abdomen for one month was suspected to have left solid adnexal tumour on ultrasound, which was later diagnosed to be ovarian torsion with infarction on MRI. Unilateral salpingo-0ophorectomy was performed for the patient. A, B) Axial T2-weighted images, without and with fat saturation, showing torsion of the left ovary, which is enlarged and heterogeneous (arrow) and is showing peripheral follicles. Normal right ovary with follicles is also seen (dashed arrow). C, D) Axial T1-weighted images without and with fat saturation, showing peripheral haemorrhagic rim in the enlarged ovary (arrows). E, F) Sagittal and coronal post-contrast T1-weighted image with fat saturation showing that the affected ovary is not enhancing, and only thin peripheral wall enhancement is present (arrow). Twisted ovarian pedicle causing beaking of the ovarian margin is also seen (arrowhead)

(repetition time msec/echo time msec 400-600/11-19), T2-weighted images (repetition time msec/echo time msec 3000-3200/80-90), T2-weighted fat saturated (FS) images, T1-weighted FS images, and T1-weighted FS images after intravenous injection of $0.1 \mathrm{mmol} / \mathrm{kg}$ of gadopentetate dimeglumine (Magnevist, Schering, Berlin, Germany) were acquired. Other imaging parameters were as follows: field of view $18-26 \mathrm{~cm}$; matrix size $192 \times 256$; section thickness $4 \mathrm{~mm}$, and intersection gap 2-4 mm.

Retrospective evaluation of the common MRI signs of ovarian torsion was done based on a literature search [4-8]. These included ovarian enlargement with or without associated lesion, twisted ovarian pedicle, ovarian haemorrhage, abnormal ovarian enhancement, and ipsilateral deviation of the uterus (Figures 1-10).

\section{Results}

The age of the patients ranged from 12 to 28 years. Nine of these patients presented with the clinical history of intermittent pelvic pain, with symptoms ranging from 15 days to five months. One patient complained of the absence of onset of menarche and was later diagnosed with Mayer-Rokitansky-Kuster-Hauser (MRKH) syndrome. In all patients, the initial evaluation had been done with US. Ovarian enlargement or complex adnexal masses 

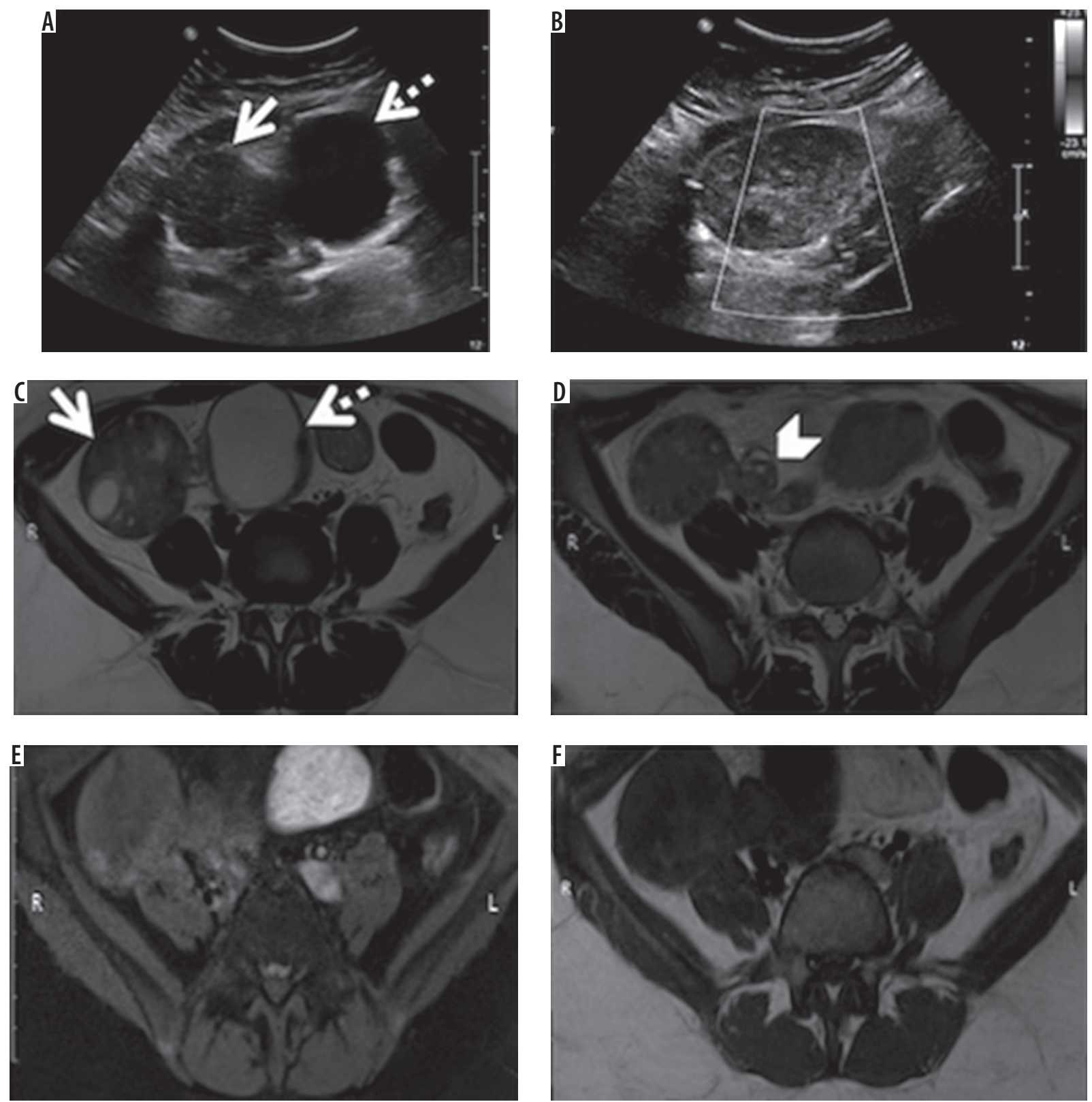

Figure 2. A 17-year-old female who complained of dull pelvic pain for 15 days, was shown to have enlarged right ovary with a paraovarian cyst on ultrasound and confirmed as torsion of the right ovary on MRI. The patient underwent laparoscopic detorsion of the ovary and is on follow-up. A, B) Ultrasound images showing an enlarged right ovary (arrow) with a paraovarian cyst (dashed arrow). The ovary did not show any internal vascularity on colour Doppler. C) Axial T2-weighted image showing slightly enlarged right ovary (arrow), with an associated approximately $4.5 \mathrm{~cm}$ paraovarian cyst (dashed arrow). D) Axial T2-weighted image showing twisted pedicle (arrowhead). E, F) Axial T1-weighted images with and without fat saturation, respectively, showing enlarged hypointense right ovary

were reported on the left side in three patients and on the right side in seven patients. Two patients had also undergone CT because a neoplastic aetiology was suspected on the US (Figures 4, 5). However, a confident diagnosis of ovarian torsion could not be made on US or CT. MRI was done to characterise these masses and confirm the diagnosis. Patients underwent laparoscopic surgery after the diagnosis of ovarian torsion was confirmed. Unilateral salpingo-oophorectomy was performed for nine patients because their ovaries were found to be non-viable intraoperatively. One patient underwent detorsion of the ovary and is stable on follow-up.

\section{Ovarian enlargement with or without associated lesion}

All patients showed an enlarged ovary, with sizes ranging from 5 to $18 \mathrm{~cm}$. Infarcted ovaries with necrosis were seen in two patients. These ovaries were observed as large complex masses with cystic components (Figures 4, 5) [6]. Peripherally lying follicles could be identified in nine cases (Figures 1-7, 9, 10). Two of the affected ovaries showed paraovarian cysts, measuring 4.5 and $3.5 \mathrm{~cm}$ in the largest diameter (Figures 2, 9). These paraovarian cysts were simple cysts without any solid component. One of the enlarged ovaries showed MRI signal characteristic of fat (T1, T2 

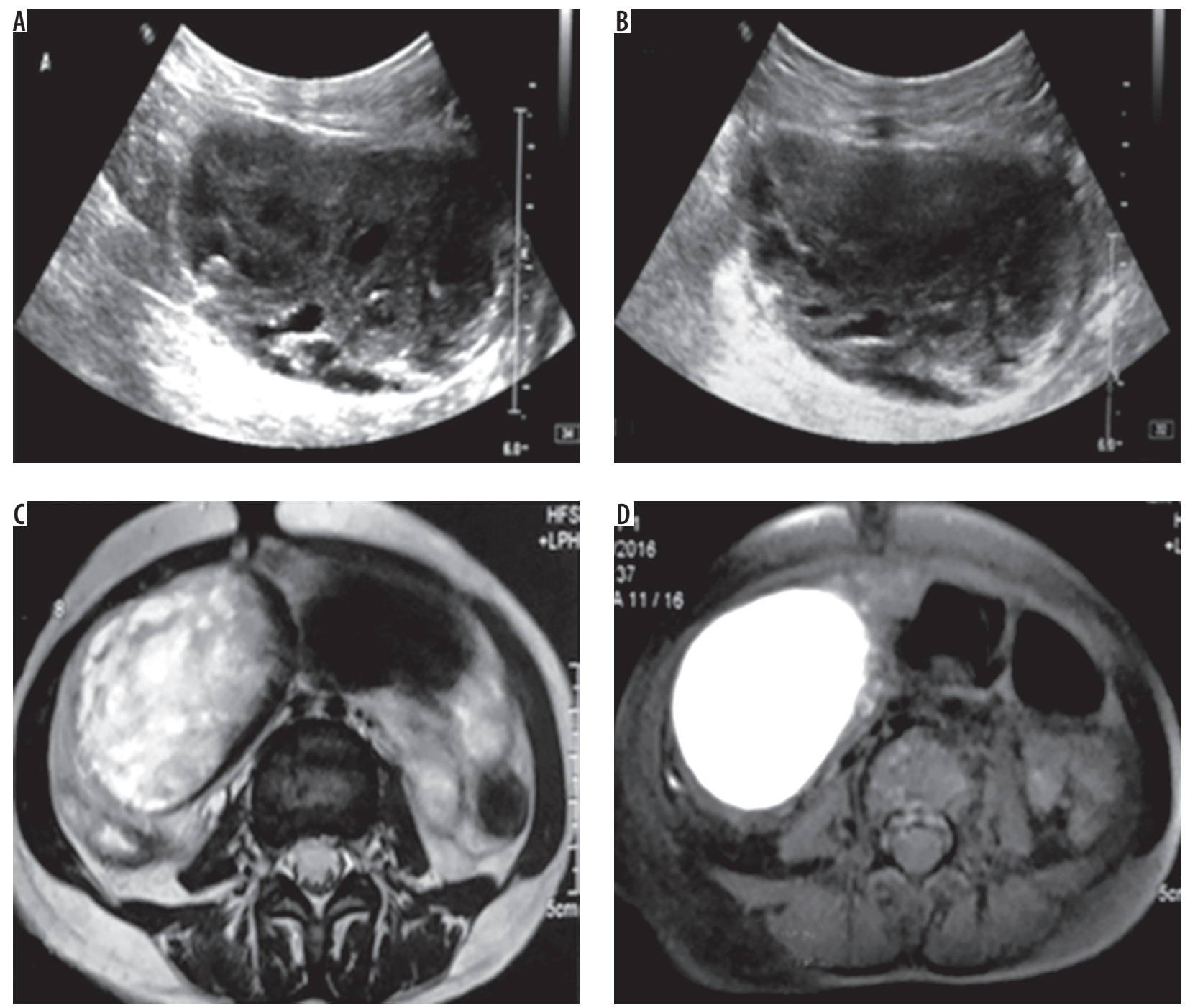

Figure 3. A 15-year-old female who complained of intermittent episodes of pelvic pain for four months, who was shown to have a large right adnexal mass on ultrasound, confirmed as torsion with complete infarction of right ovary on MRI. Unilateral salpingo-0ophorectomy was performed for the patient. A, B) Ultrasound images showing heterogeneous right adnexal mass with cystic areas seen within it. C) Axial T2-weighted image showing enlarged, heterogeneously hyperintense right ovary. D) Axial T1-weighted image with fat saturation, showing diffusely hyperintense right ovary suggestive of haemorrhage in the ovary

hyperintense signal, which was suppressed on FS images) within it (Figure 10). This lesion was reported as dermoid on MRI and was confirmed on histopathology post-surgery.

One patient showed torsion of the right ovary, ectopic left ovary, and absence of uterus on MRI (Figure 6). These features were suggestive of MRKH syndrome.

\section{Twisted ovarian pedicle}

'Twisted ovarian pedicle' sign was seen in seven patients. The fallopian tube or vascular channels were seen in helical configuration on MRI (Figures 2, 4, 6, 8-10) [2,4]. Beaking of the margin of an enlarged ovary due to the twisted pedicle was also seen (Figure 1) [7,8]. Postcontrast MRI sequences were useful to better demonstrate the twisted ovarian pedicle in some cases (Figures 1,9).

\section{Ovarian haemorrhage}

The majority of the cases (eight) showed areas of subacute haemorrhage (T1, T2 hyperintensity, non-suppression on FS sequences) within the ovarian stroma. This signal was seen predominantly in the periphery of the enlarged ovary in six cases (Figures 1, 4, 6, 8-10); while in two cases, a large part of the ovarian stroma (central as well as peripheral) showed the MRI signal of subacute haemorrhage (Figures 3,5 ). Haemorrhage was also noted within the twisted pedicle in two cases (Figures 8, 10). Haemorrhagic pedicle was associated with haemorrhage in the ovarian stroma. All patients showing ovarian haemorrhage on MRI were found to have infarcted ovaries intraoperatively. Out of two patients in whom MRI did not show ovarian haemorrhage, one was found to have a viable ovary, and detorsion was done for the same (Figure 2). 

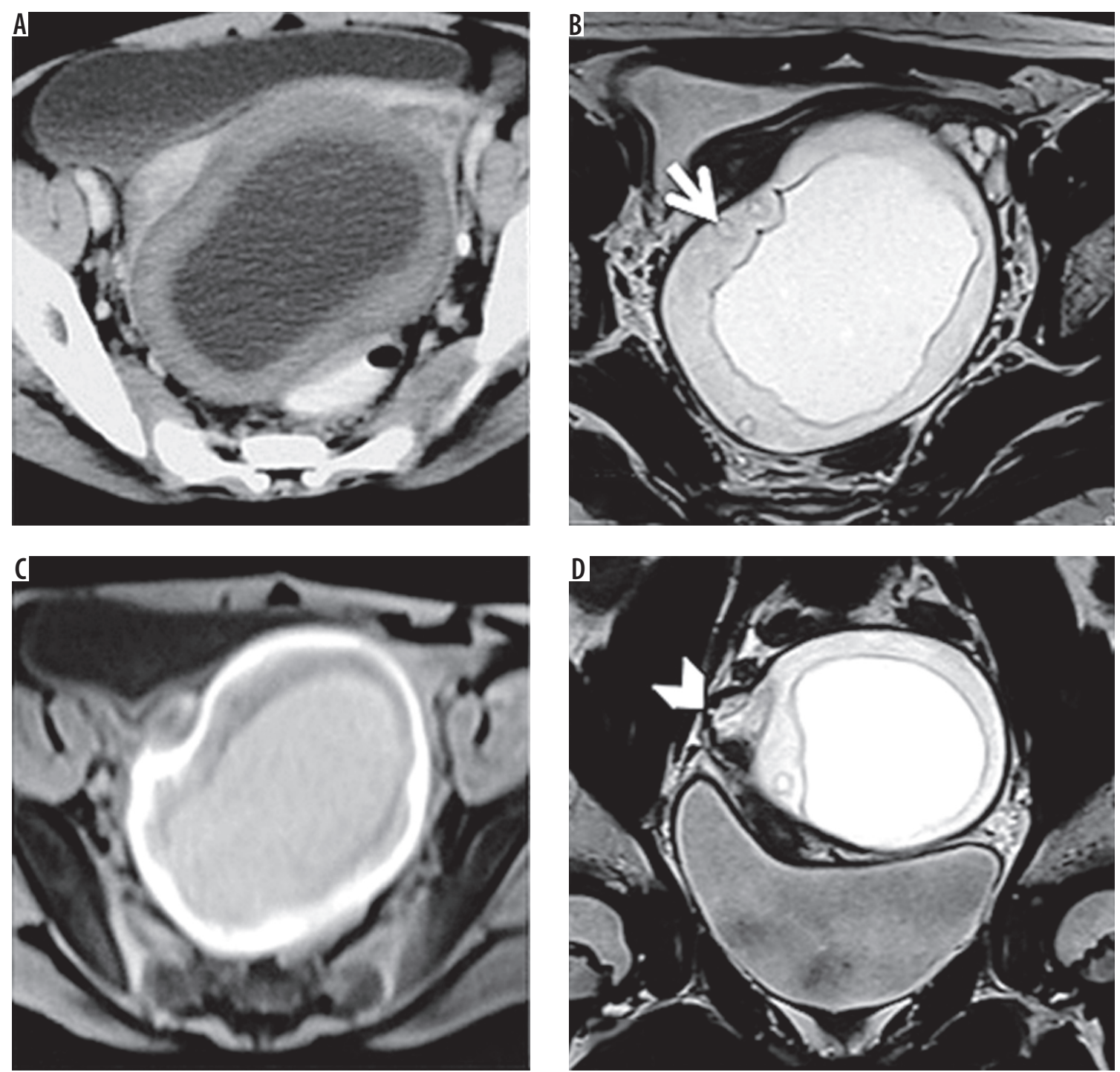

Figure 4. A 12-year-old female who complained of pelvic pain for three months, who was shown to have a large cystic mass with thick walls in right adnexa on CT, which was confirmed as torsion with infarction of right ovary on MRI. Unilateral salpingo-oophorectomy was performed for the patient. A) Axial (T image showing cystic mass with thick walls in the pelvis. B) Axial T2-weighted image showing mass with thick hyperintense walls, which shows small follicles in the periphery (arrow). C) Axial T1-weighted image showing the peripheral hyperintense signal of ovary suggestive of haemorrhage. D) Coronal T2-weighted image showing twisted pedicle (arrowhead) along the margin of the twisted right ovary

\section{Abnormal ovarian enhancement}

Non-enhancement of ovarian stroma was seen in six patients. All these patients had non-viable ovaries intraoperatively. Two cases showed a smooth or irregular peripheral enhancing wall of ovaries (Figures 1, 10). Mild ovarian stromal enhancement was seen in two cases (Figures 2, 7).

\section{Ipsilateral deviation of the uterus}

Seven patients showed ipsilateral deviation of the uterus (Figures 1, 4,7). Two patients showed contralateral deviation of the uterus due to the mass effect of the enlarged ovary (Figures 5, 9). In one patient, there was no uterus (Figure 6).

\section{Other findings}

A small amount of free fluid in the pelvis was seen in four patients (Figure 6). None of the patients showed haemoperitoneum.

\section{Discussion}

\section{Ovarian enlargement with or without associated lesion}

Ovarian enlargement, although non-specific, is the most common finding in patients with ovarian torsion $[4,5]$. Enlarged ovary with peripherally displaced follicles and central afollicular stroma is a more specific sign of ovarian torsion. Massive ovarian oedema refers to tumour-like marked enlargement of the ovary secondary to intermittent 

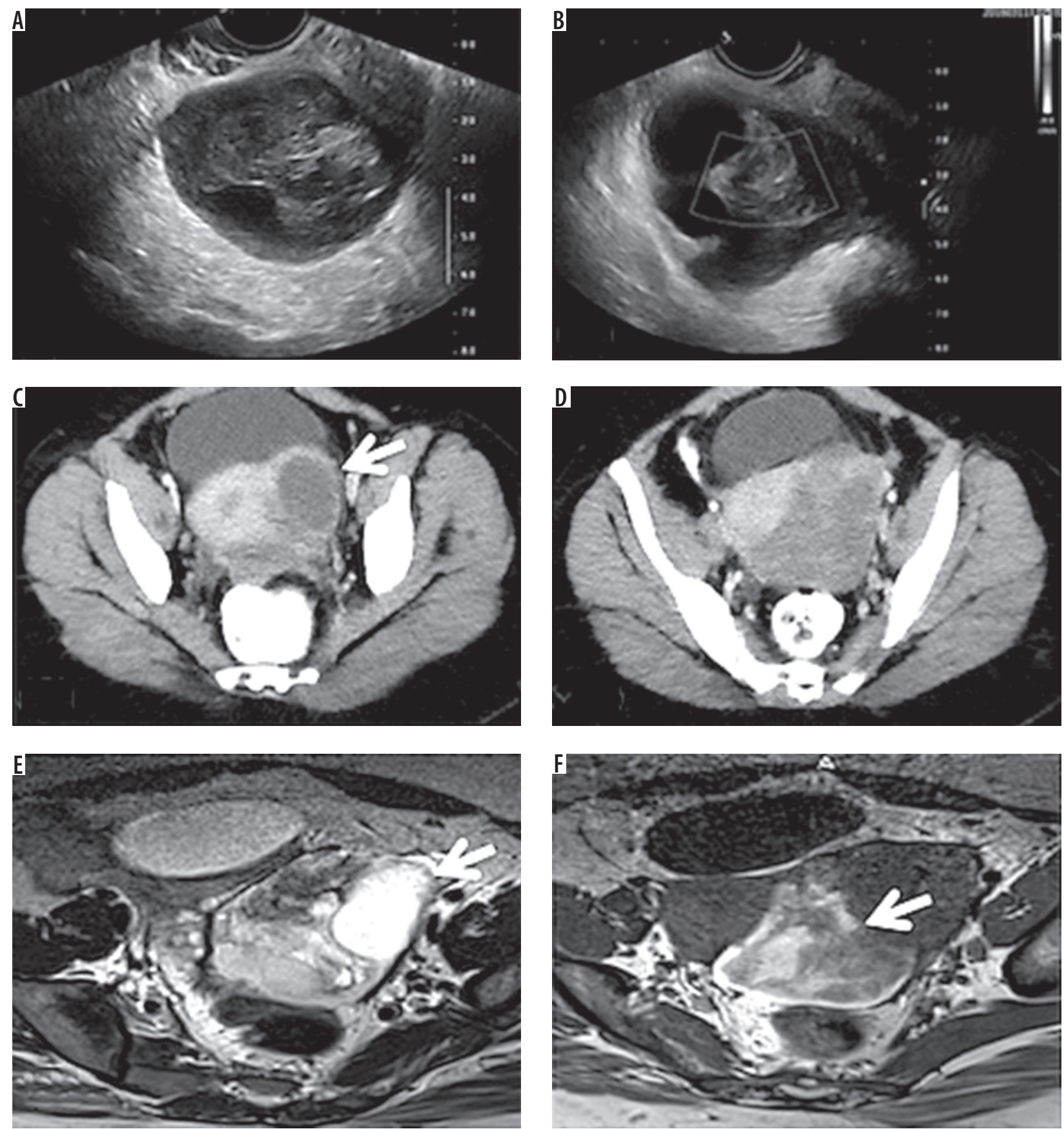

Figure 5. A 28-year-old female who complained of pelvic pain for five months, who was shown to have an adnexal mass on ultrasound and CT, confirmed as torsion with infarction of the left ovary on MRI. Unilateral salpingo-0ophorectomy was performed for the patient. A, B) Ultrasound images showing heterogeneous mass with cystic areas in the left adnexa, showing no internal vascularity on colour Doppler. C, D) Axial CT images showing heterogeneously hypodense mass in the region of left adnexa, with an area of cystic attenuation (arrow). E) Axial T2-weighted image showing enlarged and heterogeneously hyperintense left ovary, with an enlarged follicular cyst (measuring approximately $3.2 \mathrm{~cm}$ ) in its periphery (arrow). F) Axial T1-weighted image showing the hyperintense signal of ovarian stroma suggestive of haemorrhage (arrow)

torsion, which leads to extensive stromal oedema without infarction. It shows enhancement of stroma on MRI as opposed to infarction. Untreated ovarian torsion may lead to infarction with necrosis, which appears as an enlarged adnexal mass with cystic components on MRI and shows absent enhancement on postcontrast sequences [6].

Lesions that may be seen in association with ovarian torsion include follicular cysts, haemorrhagic cysts, corpus luteal cysts, paraovarian cysts, and benign and malignant neoplasms. Dermoids are the most common neoplasms identified in ovaries with torsion [3]. They can be identified by their fat content, which is seen as hyperintense signal on $\mathrm{T} 1$ and $\mathrm{T} 2$ sequences and suppressed on FS images.

Enlarged ovary may also be seen in ovarian hyperstimulation syndrome, but clinical history, bilaterality, and the presence of extravascular fluid collections will help to diagnose this condition. However, torsion can also occur 

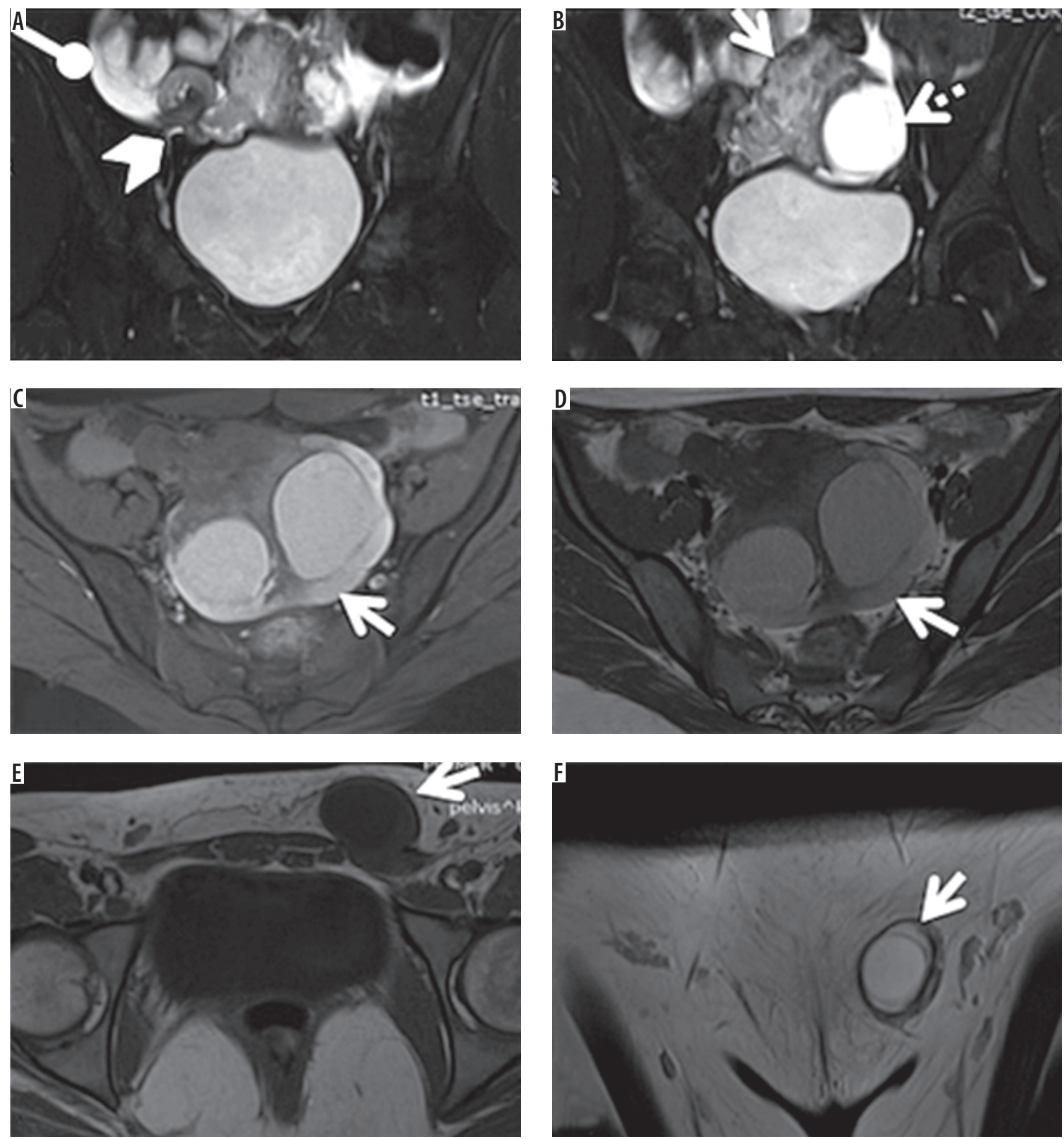

Figure 6. An 18-year-old girl, who complained of the absence of onset of menarche, was found to have an absent uterus and a large pelvic mass on ultrasound. Torsion of the right ovary, absent uterus (Mayer-Rokitansky-Kuster-Hauser syndrome), and a left ectopic ovary were diagnosed on MRI. The patient underwent unilateral salpingo-0ophorectomy. A, B) Coronal T2-weighted images with fat saturation showing torsion of right ovary, which was enlarged and heterogeneous (arrow), with twisted pedicle seen along its lateral margin (arrowhead), and a few enlarged follicles seen in its periphery (dashed arrow). Ascites was also seen (round tip arrow). C, D) Axial T1-weighted images with and without fat saturation, showing haemorrhagic follicle cysts with haemorrhage in the ovarian stroma (arrows). E, F) Axial T1-weighted image, and coronal T2-weighted image showing left ectopic ovary with follicles in the left inguinal canal (arrows)

in hyperstimulated ovaries, which can be identified if they show asymmetrical enlargement, abnormal enhancement, haemorrhage, and twisted ovarian pedicle.

One of the cases of ovarian torsion reported by us was in a patient with absent uterus, diagnosed as MRKH syndrome. The association of ovarian torsion with $\mathrm{MRKH}$ syndrome has been reported previously, and the absence of supporting structures of ovary, primarily the utero-ovarian ligament, has been postulated to be the cause [9].

\section{Twisted ovarian pedicle}

The twisted ovarian pedicle is a pathognomonic sign of ovarian torsion [4]. It is formed due to the twisting of the 

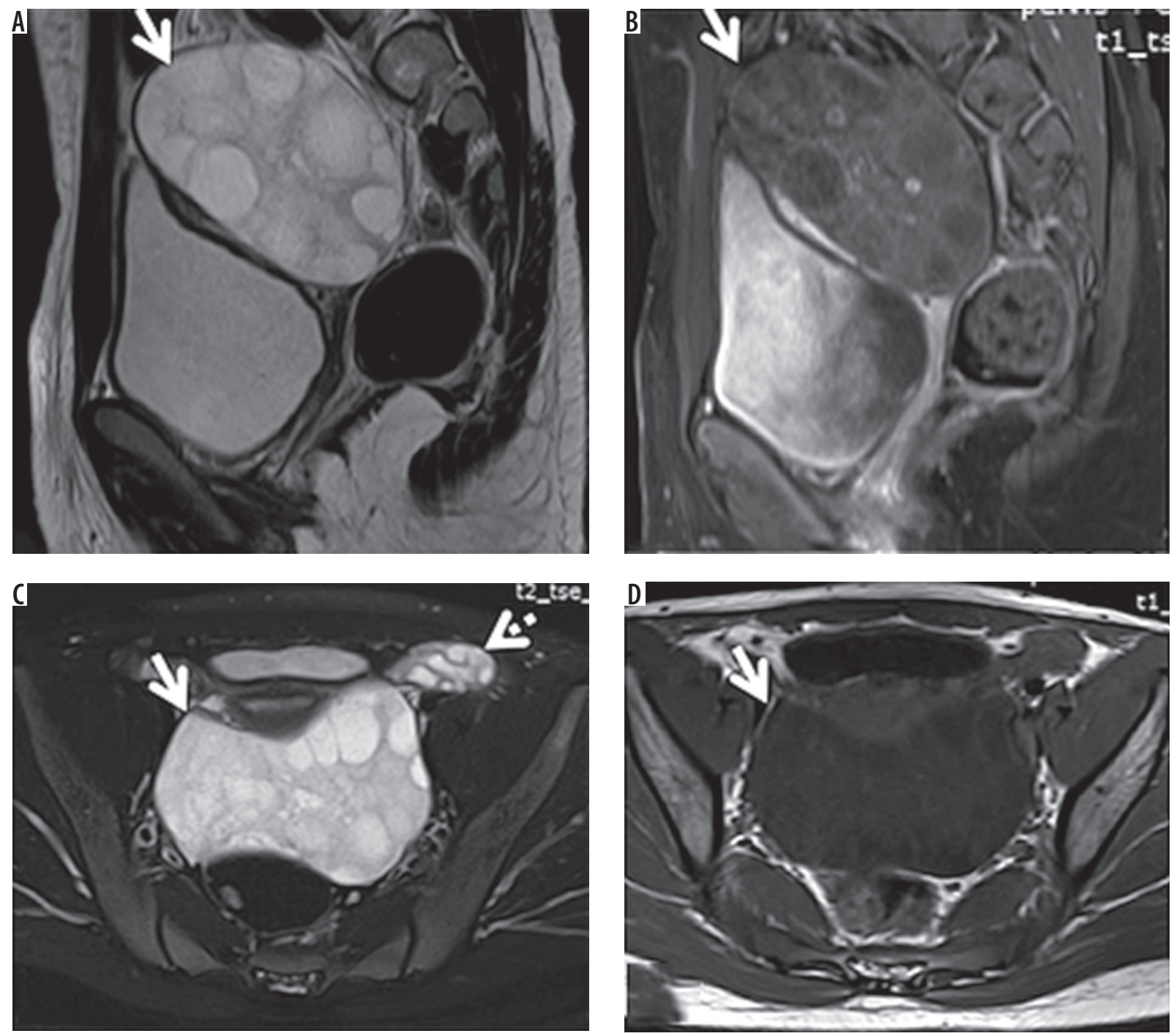

Figure 7. A 12-year-old female who presented with pelvic pain for two months, diagnosed with torsion of the right ovary on MRI. Unilateral salpingooophorectomy was performed because the ovary was found to be non-viable intraoperatively. A) Sagittal T2-weighted image showing the right ovary, which is diffusely enlarged and heterogeneously hyperintense, showing small to enlarged follicles (arrow). B) Sagittal post-contrast T1-weighted image with fat saturation showing minimal enhancement of enlarged ovary (arrow). C) Axial T2-weighted image with fat saturation showing enlarged right ovary (arrow) and normal sized left ovary (dashed arrow). D) Axial T1-weighted image showing an enlarged hypointense right ovary (arrow)

broad ligament containing the thickened fallopian tube and vascular channels [2]. Fallopian tube or broad ligament comprising vascular channels are seen in helical configuration on MRI $[2,4]$. It is essential to evaluate the images in all three planes to identify the twisted ovarian pedicle. Postcontrast MRI sequences are also useful in demonstrating the twisted ovarian pedicle.

Isolated fallopian tube torsion without ovarian torsion can also show thick and twisted pedicle. However, the ipsilateral normal ovary will be seen along with it. Isolated fallopian tube torsion also requires surgical intervention.

\section{Ovarian haemorrhage}

Ovarian haemorrhage is highly associated with ovarian torsion with infarction [10-14]. MRI is a sensitive imaging modality for identifying haemorrhage. Subacute haemorrhage is seen as a hyperintense signal on both T1 and T2 sequences, not suppressed on FS images. Haemorrhage may be seen in the periphery of the enlarged ovary or involving the central ovarian stroma. Haemorrhage within the twisted pedicle or haemoperitoneum in the pelvis may also be seen secondary to haemorrhagic infarction of the ovary [10].

Other differential diagnoses of haemorrhagic ovarian lesions include corpus luteal cysts, haemorrhagic primary ovarian tumours or metastasis. However, lack of enhancement and the presence of twisted ovarian pedicle can help to differentiate ovarian torsion from these entities $[4,14]$.

\section{Abnormal enhancement of ovarian stroma}

Normal enhancement may be seen in the early stage of ovarian torsion or intermittent torsion. However, infarcted ovaries will show heterogeneous, minimal to absent enhancement $[8,15]$. They may show only peripheral enhancement. The presence of enhancement does not rule 

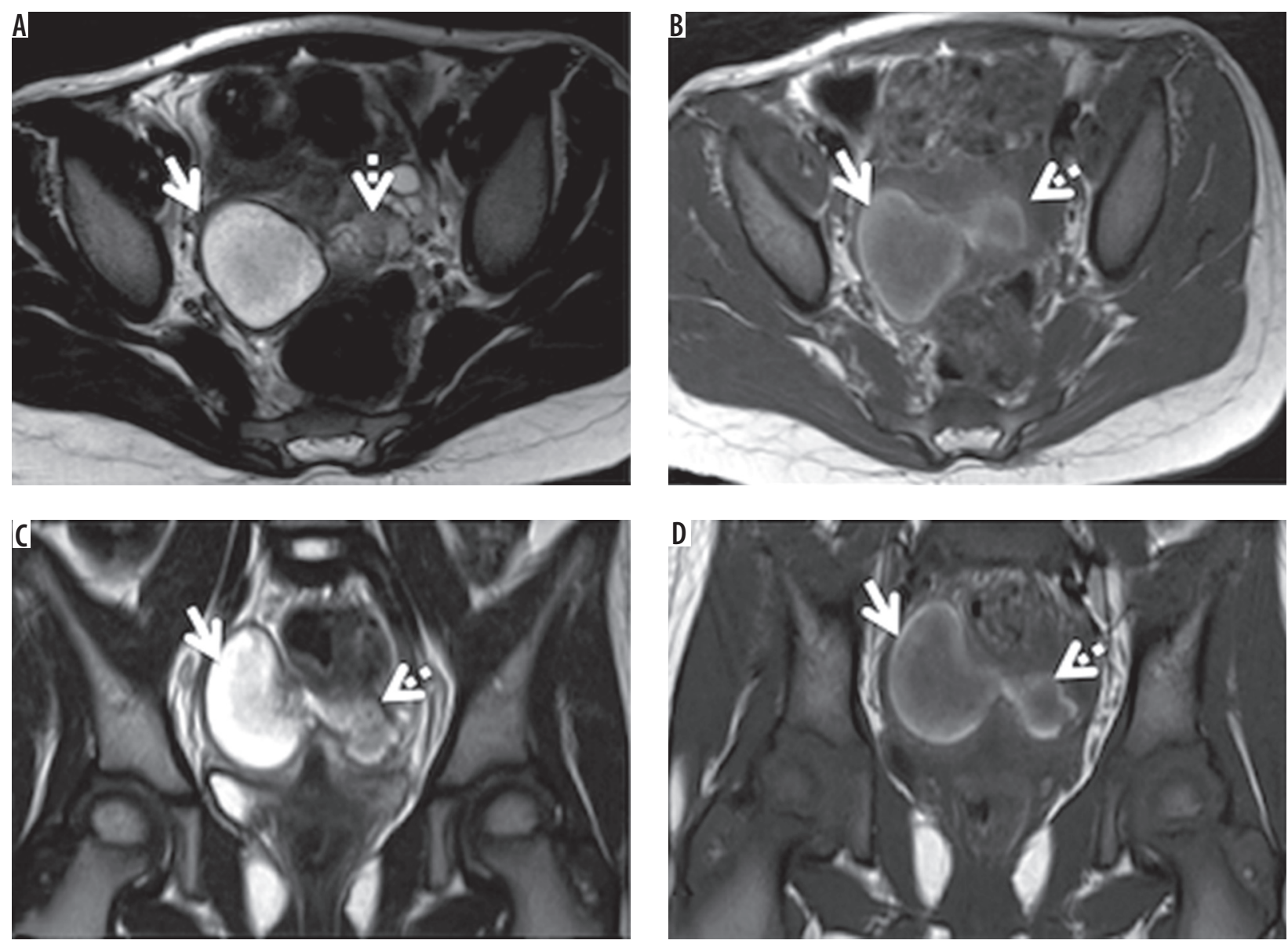

Figure 8. A 13-year-old female with pelvic pain for one month, diagnosed with torsion with infarction of right ovary on MRI. Unilateral salpingo-0ophorectomy was performed for the patient. A, C) Axial and coronal T2-weighted images showing the right ovary, which is enlarged and hyperintense (arrows), along with twisted pedicle seen along its left lateral aspect (dashed arrows). B, D) Axial and coronal T1-weighted images showing twisted right ovary (arrows) with pedicle (dashed arrows), which shows hyperintense signal suggestive of haemorrhage

out torsion; however, absent enhancement in an enlarged ovary usually indicates infarction $[4,10]$.

\section{Ipsilateral deviation of the uterus}

Ipsilateral deviated uterus seen in association with an enlarged ovary may be a useful feature for recognising ovarian torsion in some cases $[5,10,14]$. Shortening of the ipsilateral supporting structures has been postulated as the cause. However, it is not a sensitive sign of ovarian torsion. Sometimes, the contralateral deviation may also be seen due to the mass effect of the enlarged ovary.

\section{Other findings}

Chiou et al. [5] showed the presence of ascites on MRI in $50 \%$ of cases of ovarian torsion. However, it is not a sensitive or specific sign of ovarian torsion. Ascites may also be seen in association with malignant or benign adnexal tumours.

The limitations of our study include mainly its retrospective nature. Second, the small number of patients included in the study. Third, the utility of MRI in differentiating viable versus non-viable ovaries post torsion could not be evaluated. This evaluation could not be done because the majority of our cases were of non-viable (infarcted) ovaries due to the fact that our institute is a tertiary care hospital with many cases being referrals from other hospitals for complicated or undiagnosed cases. However, irrespective of ovarian viability, any ovarian torsion has to be surgically treated (detorsion or oophorectomy) [16].

\section{Conclusions}

MRI features of ovarian torsion include ovarian enlargement with or without associated lesion, twisted ovarian pedicle, ovarian haemorrhage, abnormal ovarian enhancement, and ipsilateral deviation of the uterus. Knowledge of these imaging features will enable the radiologist to recognise ovarian torsion and differentiate it reliably from other benign or malignant ovarian masses even if the clinical and ultrasound signs are inconclusive. 

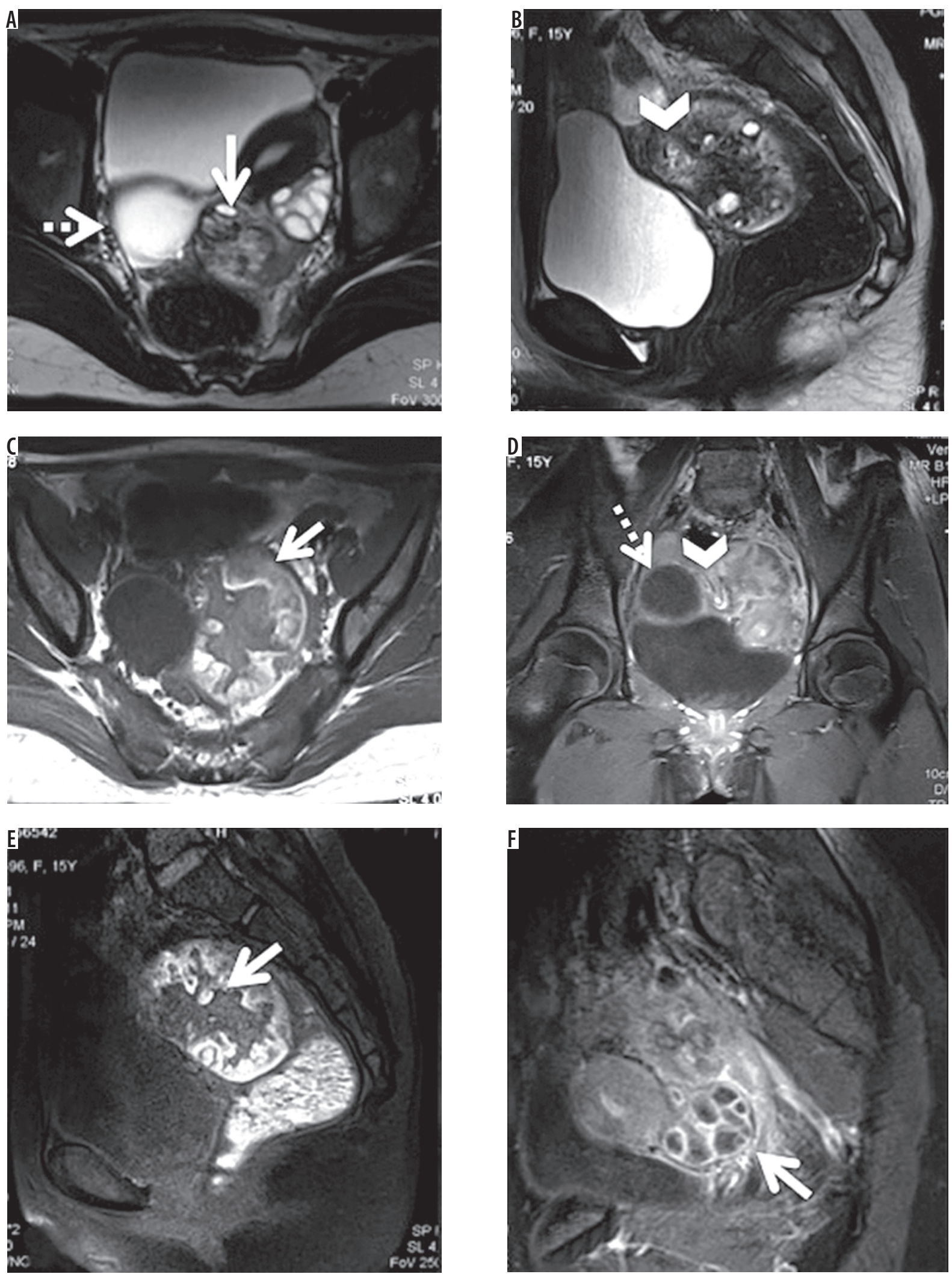

Figure 9. A 15-year-old female with pain for 15 days, diagnosed with torsion with infarction of right ovary on MRI. Unilateral salpingo-0ophorectomy was performed for the patient. A) Axial T2-weighted image showing torsion of the right ovary, which is enlarged and heterogeneous (arrow), with an associated paraovarian cyst of $3.5 \mathrm{~cm}$ diameter (dashed arrow). Normal left ovary with follicles is also seen. B) Sagittal T2-weighted image showing twisted pedicle (arrowhead). C) Axial T1-weighted image showing thick peripheral haemorrhagic rim in the ovary (arrow). D) Coronal post contrast T1-weighted image with fat saturation showing twisted pedicle (arrowhead), peripheral enhancing paraovarian cyst (dashed arrow). E) Sagittal post-contrast T1-weighted image with fat saturation showing enlarged ovary, which is not enhancing (arrow). F) Sagittal post-contrast T1-weighted image with fat saturation showing normal enhancing left ovary with follicles (arrow) 

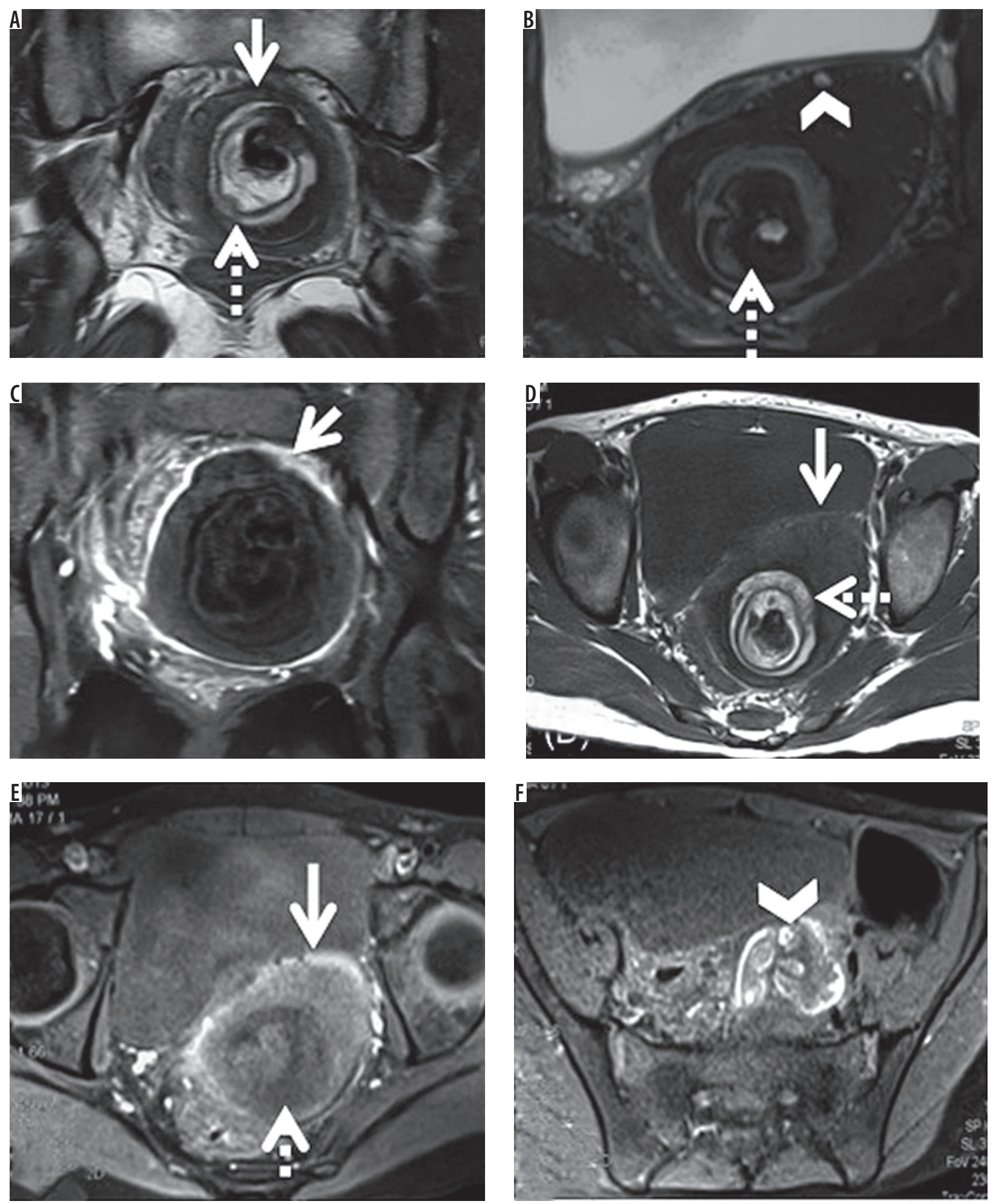

Figure 10. A 12-year-old female with pain for two months, diagnosed with torsion of left ovary with mature teratoma on MRI. Unilateral salpingo-00phorectomy with the removal of the tumour was done. A) Coronal T2-weighted image showing an enlarged ovary (arrow) with hyperintense central part (dashed arrow). B) Axial T2-weighted image with fat saturation showing suppression of signal of fat component of teratoma (dashed arrow). Small peripheral follicles are seen (arrowhead). C) Coronal post contrast T1-weighted image with fat saturation showing enlarged ovary with peripheral enhancement (arrow). D) Axial T1-weighted image showing torsion of the left ovary, which is enlarged and heterogeneous (arrow), with associated hyperintense area seen in its central part (dashed arrow). E) Axial T1-weighted image with fat saturation showing enlarged ovary with peripheral haemorrhagic rim (arrow), with hypointense area in its central part (dashed arrow), due to fat suppression. F) Axial T1-weighted image with fat saturation showing thickened twisted pedicle with haemorrhage in it (arrowhead) 


\section{Conflict of interest}

The authors report no conflict of interest.

\section{References}

1. Smolinski SE, Kreychman A, Catanzano T. Ovarian torsion: multimodality review of imaging characteristics. J Comput Assist Tomogr 2015; 39: 922-924.

2. Ghonge NP, Lall C, Aggarwal B, Bhargava P. The MRI whirlpool sign in the diagnosis of ovarian torsion. Radiol Case Rep 2015; 7: 731.

3. Hiller N, Appelbaum L, Simanovsky N, et al. CT features of adnexal torsion. Am J Roentgenol 2007; 189: 124-129.

4. Duigenan S, Oliva E, Lee SI. Ovarian torsion: diagnostic features on CT and MRI with pathologic correlation. AJR Am J Roentgenol 2012; 198: W122-131.

5. Chiou SY, Lev-Toaff AS, Masuda E, et al. Adnexal torsion: new clinical and imaging observations by sonography, computed tomography, and magnetic resonance imaging. J Ultrasound Med 2007; 26 1289-1301.

6. Ssi-Yan-Kai G, Rivain AL, Trichot C, et al. What every radiologist should know about adnexal torsion. Emerg Radiol 2018; 25: 51-59.

7. Wilkinson C, Sanderson A. Adnexal torsion - a multimodality imaging review. Clin Radiol 2012; 67: 476-483.

8. Lourenco AP, Swenson D, Tubbs RJ, et al. Ovarian and tubal torsion: imaging findings on US, CT, and MRI. Emerg Radiol 2014; 21: $179-187$.
9. Brown PF, McLendon HL, Pettitt BJ. Ovarian torsion in a patient with congenital absence of the uterus. Pediatr Surg Int 1992; 7: 67-68.

10. Rha SE, Byun JY, Jung SE, et al. CT and MR imaging features of adnexal torsion. RadioGraphics 2002; 22: 283-294.

11. Kawakami K, Murata K, Kawaguchi N, et al. Hemorrhagic infarction of the diseased ovary: a common MR finding in two cases. Magn Reson Imaging 1993; 11: 595-597.

12. Moribata Y, Kido A, Yamaoka T, et al. MR imaging findings of ovarian torsion correlate with pathological hemorrhagic infarction. J Obstet Gynaecol Res 2015; 41: 1433-1439.

13. Petkovska I, Duke E, Martin DR, et al. MRI of ovarian torsion: Correlation of imaging features with the presence of perifollicular hemorrhage and ovarian viability. Eur J Radiol 2016; 85: 2064-2071.

14. Kimura I, Togashi K, Kawakami S, et al. Ovarian torsion: CT and MR imaging appearances. Radiology 1994; 190: 337-341.

15. Iraha Y, Okada M, Iraha R, et al. CT and MR imaging of gynecologic emergencies. Radiographics 2017; 37: 1569-1586.

16. Patil AR, Nandikoor S, Rao A, et al. Multimodality imaging in adnexal torsion. J Med Imaging Radiat Oncol 2015; 59: 7-19. 